\title{
Diagnóstico, Conduta Obstétrica e Resultados Perinatais em Fetos com Hidrocefalia
}

\author{
Diagnosis, Obstetrical Management and Perinatal Outcome in Hydrocephalus
}

Renato Passini Júnior, Simone Pimenta Nóbrega, José Guilherme Cecatti Ricardo Barini, João Luiz Pinto e Silva

\section{RESUM0}

\begin{abstract}
Objetivo: avaliar os métodos para diagnóstico, características da gestação, complicações maternas e resultados perinatais em casos de hidrocefalia congênita, correlacionando-os com variáveis gestacionais e do parto.

Método: avaliaram-se 116 gestações com este diagnóstico antes ou após o parto, dos quais 112 ocorridos na Maternidade do CAISM da UNICAMP no periodo de 1986 a 1995. Para as variáveis perinatais, utilizaram-se os dados completos de 82 recém-nascidos. Para a análise dos dados, calcularam-se as distribuições e médias, usando-se os testes de $c^{2}$ e exato de Fisher.

Resultados: geralmente o diagnóstico foi anterior ao parto, confirmado pelo exame ecográfico, e o parto foi por cesárea na maioria dos casos. A cefalocentese foi realizada em 11 casos $e$ as complicações no parto vaginal foram mais freqüentes que na cesárea. $O$ índice de Apgar baixo foi mais freqüente entre os recém-nascidos de parto vaginal. A hidrocefalia congênita esteve também associada a importante morbidade e mortalidade neonatal e perinatal, outras malformações e número muito pequeno de crianças sem seqüelas.

Conclusões: a avaliação destes fatores pode ser de grande utilidade para o obstetra acompanhar gestantes com esta malformação fetal, dando maior respaldo às decisões que, além de médicas e éticas, devem levar em conta a relação risco-benefício das medidas a serem tomadas.
\end{abstract}

PALAVRAS-CHAVE: Hidrocefalia. Diagnóstico pré-natal. Malformações fetais. Ultrasonografia.

Introdução

A existência de malformação congênita diagnosticada durante a gestação é uma grande preocupação, tanto para pais quanto para obstetras. Aquelas relacionadas ao Sistema Nervoso

Departamento de Tocoginecologia, Faculdade de Ciências Médicas, Universidade Estadual de Campinas Correspondência:

Renato Passini Júnior

CAISM - UNICAMP

Caixa Postal 6030

13081-970 Campinas - SP
Central assumem grande importância, não só pela sua freqüência e letalidade (em casos como anencefalia e/ou acrania), mas também pelo número e gravidade de seqüelas que podem causar, prejudicando a evolução da criança. Dessas malformações, a mais freqüente é a hidrocefalia, cuja incidência é próxima de 1/2.000 partos, compreendendo $12 \%$ de todas as malformações graves encontradas por ocasião do parto ${ }^{7}$.

A etiologia pode envolver distúrbios genéticos como obstrução do aqueduto de Sylvius, síndrome de Dandy-Walker, malformação de Arnold Chiari, agenesia cerebelar e espinha bífida ${ }^{9}$, até quadros infecciosos, hemorragia intracraniana, ou mesmo 
não ser identificada ${ }^{7}$. Dentre os quadros infecciosos, destacam-se a toxoplasmose, presente em 10,6\% dos casos ${ }^{8}$, a citomegalovirose e a sífilis ${ }^{10}$, que são exemplos de condições que podem provocar obstruções ao fluxo liquórico ou interferir na sua produção e drenagem, levando ao acúmulo de líquido céfalorraquidiano, dilatação ventricular e aparecimento do quadro clínico de hidrocefalia.

Estima-se que aproximadamente 52\% dos casos são diagnosticados ainda durante a gravidez, possivelmente pelo uso sistemático de exames ultra-sonográficos ${ }^{2}$. Além dessa avaliação, têm-se indicado o estudo genético do feto, que se tornou possível com o desenvolvimento de técnicas de punção do cordão umbilical (cordocentese) e coleta de sangue para análise de cariótipo. Entretanto, alguns casos são diagnosticados posteriormente, ou no momento do parto, por dificuldade na progressão fetal ${ }^{7,12}$, durante o exame do recémnascido quando existe a macrocrania, ou no período neonatal ao se realizar propedêutica especializada como transiluminação, ultrasonografia, eletroencefalograma ou tomografia computadorizada ${ }^{10}$.

Buscando reduzir o volume craniano e minorar eventuais lesões cerebrais, que são mais freqüentes quando o parênquima cerebral é reduzido a uma espessura menor que $20 \mathrm{~mm}$, são descritas na literatura estudos abordando punções intraventriculares (cefalocentese) únicas, seriadas ou com a colocação de cateteres de drenagem contínua ${ }^{7}$. Entretanto, não existe consenso de que esse procedimento melhore o prognóstico das crianças $^{16}$.

Para o obstetra, o acompanhamento de gestações com essa anomalia é sempre complexo, pois é freqüente a associação com outras condições que agravam seu desenvolvimento, como o oligoâmnio e polidrâmnio ${ }^{17}$, apresentação pélvica e maior risco de rotura uterina ${ }^{7}$.

Um diagnóstico de hidrocefalia desencadeia, além de um drama psicológico familiar, uma série de questionamentos para o obstetra, que se vê entre a dificuldade de encontrar a causa da patologia e a de estabelecer um prognóstico futuro, quase sempre muito difícil. As crianças com hidrocefalia exigem grandes cuidados, particularmente no período neonatal. Geralmente são recém-nascidos com peso e estatura abaixo da média para a idade gestacional, podendo nascer com baixa vitalidade pelas dificuldades no parto ${ }^{17}$.

Dependendo da gravidade do quadro, muitas necessitarão de internação prolongada em Unidade de Terapia Intensiva (UTI) neonatal, principalmente se houver prematuridade associada, acarretando a necessidade de uma série de medidas terapêuticas e condutas complexas, de alto custo econômico ${ }^{11}$. Em um estudo, incluindo 50 casos de hidrocefalia congênita, $22 \%$ das crianças morreram no primeiro ano de vida, $6 \%$ no período neonatal tardio e $4 \%$ após 28 dias, com sobrevida de apenas $28 \%$ ao final do acompanhamento ${ }^{4}$.

Atualmente o único consenso é que o prognóstico é tanto mais grave, quanto mais precocemente surge a hidrocefalia no feto ${ }^{14}$. Entretanto, vários fatores devem ser analisados, como velocidade de progressão ${ }^{18}$, etiologia ${ }^{10}$, associação com outras malformações ${ }^{15}$, complicações durante o parto ${ }^{12}$, complicações neonatais ${ }^{11}$ e espessura cortical ao nascimento.

A indefinição do prognóstico também dificulta condutas obstétricas como a avaliação da vitalidade fetal $^{1}$, a definição do melhor momento para o parto ${ }^{11}$ e sua via mais adequada ${ }^{3}$. Quanto ao melhor momento para interromper a gestação, é aconselhável, sempre que possível, aguardar a maturidade fetal $^{7}$. A definição da via de parto depende das dimensões do pólo cefálico e do prognóstico fetal.

Discutem-se num estudo ${ }^{5}$, à luz da ética, estratégias de resolução da gravidez para os extremos de hidrocefalia isolada. Nesses casos, a via de parto escolhida foi a cesárea, por acarretar menor tocotraumatismo ao feto, possibilitando maior sucesso no tratamento posterior. A cefalocentese redutora, seguida de parto vaginal, ficou reservada para situações de hidrocefalia fetal associada a anormalidades incompativeis com a vida, pois a punção liquórica acarreta o risco de atingir os vasos cerebrais e provocar hemorragia intracraniana ${ }^{13,18}$.

Entretanto, sabe-se também que, mesmo na cesariana, pode haver grande dificuldade para a retirada do pólo cefálico, obrigando à realização de manobras mais agressivas que podem comprometer a vitalidade do recém-nascido. Além disso, existe a possibilidade de lesões de tecidos maternos, com complicações hemorrágicas intraparto, podendo levar à histerectomia e comprometimento do futuro obstétrico da paciente ${ }^{7}$.

O objetivo do presente estudo foi avaliar os métodos para diagnóstico, características da gestação, complicações maternas e resultados perinatais em casos de hidrocefalia congênita, correlacionando-os com variáveis gestacionais e do parto.

\section{Pacientes e Métodos}

Este é um estudo descritivo retrospectivo, no qual foram avaliadas as gestações com diagnóstico de hidrocefalia fetal e/ou neonatal congênita, com 
partos ocorridos entre 1986 e 1995 na Maternidade do Centro de Atenção Integral à Saúde da Mulher, Departamento de Tocoginecologia da Faculdade de Ciências Médicas da Universidade Estadual de Campinas (UNICAMP).

As informações foram coletadas de fichas obstétricas e fichas neonatais pré-codificadas, de prontuários médicos, e transcritas para uma ficha criada para esse fim, procedendo-se à revisão das mesmas. Os dados foram digitados utilizando-se um banco de dados criado no programa Epi-Info.

Foram incluídos no estudo todos os casos de gestações com recém-nascidos com hidrocefalia congênita, independente da época de diagnóstico ou da condição de nascimento da criança. Estudaram-se variáveis relativas à gestante, à gestação, diagnóstico da hidrocefalia, parto, complicações e resultados perinatais.

Os dados foram analisados através das freqüências de variáveis, cálculo de médias, desvios-padrão, teste do $\chi^{2}$ e Exato de Fisher. Não houve identificação das mães ou crianças. Garantiu-se, portanto, total sigilo da fonte das informações.

\section{Resultados}

A idade das 116 mulheres identificadas variou de 15 a 41 anos, com média de 24,8 anos. A paridade variou de zero até 6 partos, com média de 1,5 . Os antecedentes obstétricos patológicos foram encontrados em $42,2 \%$ das pacientes (Tabela 1). Do total de mulheres, 25\% (23 casos) eram sabidamente fumantes e 2,9\% (3 casos) eram alcoólatras. O antecedente de malformação foi positivo em 9 casos, representando $10,8 \%$.

Em relação a drogas e/ou medicamentos, 16 das gestantes estudadas $(13,8 \%)$ referiram seu uso durante a gestação. Houve 3 casos em que foi usada alfa-metildopa, 2 terbutalina e penicilina e 1 misoprostol, verapamil, benzodiazepínico, ampicilina, organofosforado, corticosteróide, macrodantina, digoxina e sulfametoxazol/trimetropim.

Cerca de 6\% dessas gestantes não fizeram pré-natal. Dentre as que fizeram, 20\% não sabia informar o número de consultas. A média de consultas entre as informantes foi de 5,3. Patologias relacionadas ou não com a gestação ocorreram em $43,5 \%$ das pacientes durante a gestação estudada.

A maior parte das gestantes $(78,4 \%)$ teve o diagnóstico de hidrocefalia realizado durante a gravidez. Cabe ressaltar que o diagnóstico prénatal foi ecográfico em $72,4 \%$ dos casos e realizado em média na $31^{\mathrm{a}}$ semana de gestação. O diagnóstico ecográfico da hidrocefalia durante a gravidez não influenciou de modo significativo a forma de início do trabalho de parto, embora tenha aumentado o número de partos induzidos, nem alterou a via de parto (Tabela 2). O parto foi cesariana em cerca de $63 \%$ dos casos (42 de 112).

Tabela 1 - Distribuição dos casos com hidrocefalia congênita, segundo algumas características das mulheres e antecedentes obstétricos.

\begin{tabular}{|c|c|c|}
\hline Características & $\mathbf{n}$ & $\%$ \\
\hline \multicolumn{3}{|l|}{ Idade (anos) } \\
\hline 15 a 19 & 24 & 20,7 \\
\hline 20 a 24 & 39 & 33,6 \\
\hline 25 a 29 & 21 & 18,1 \\
\hline 30 a 34 & 24 & 20,7 \\
\hline$\geq 35$ & 8 & 6,9 \\
\hline Total & 116 & 100,0 \\
\hline \multicolumn{3}{|l|}{ Paridade } \\
\hline Nuliparas & 33 & 28,4 \\
\hline 1 & 36 & 31,0 \\
\hline 2 & 22 & 19,0 \\
\hline$\geq 3$ & 25 & 21,5 \\
\hline Total & 116 & 100,0 \\
\hline \multicolumn{3}{|c|}{ Antecedentes obstétricos } \\
\hline Aborto espontâneo & 14 & 16,8 \\
\hline Aborto de repetição & 8 & 9,6 \\
\hline TPP & 6 & 7,2 \\
\hline $\mathrm{DPP}$ & 2 & 2,4 \\
\hline Pós-termo & 1 & 1,2 \\
\hline Hemorragia & 1 & 1,2 \\
\hline Isoimunização Rh & 1 & 1,2 \\
\hline Óbito fetal & 1 & 1,2 \\
\hline Pré-eclâmpsia & 1 & 1,2 \\
\hline Antecedente & 35 & 42,2 \\
\hline Total & 85 & 100,0 \\
\hline
\end{tabular}

TPP - Trabalho de parto prematuro

DPP - Descolamento prematuro de placenta

Tabela 2 - Forma de início e término do parto em casos de feto com hidrocefalia congênita segundo diagnóstico ecográfico durante a gravidez.

\begin{tabular}{|c|c|c|c|}
\hline \multirow[t]{2}{*}{ Parto } & \multicolumn{2}{|c|}{ Diagnóstico pré-natal ecográfico } & \multirow[b]{2}{*}{ Total $^{\mathrm{a}}$} \\
\hline & Sem & Com & \\
\hline \multicolumn{4}{|l|}{ Forma de início ${ }^{b}$} \\
\hline Espontâneo & 18 & 31 & 49 \\
\hline Induzido & 1 & 8 & 9 \\
\hline Cesárea eletiva & 13 & 41 & 54 \\
\hline Total & 32 & 80 & 112 \\
\hline \multicolumn{4}{|c|}{ Forma de término ${ }^{c}$} \\
\hline Vaginal & 14 & 28 & 42 \\
\hline Cesárea & 18 & 52 & 70 \\
\hline Total & 32 & 80 & 112 \\
\hline
\end{tabular}

A realização de cefalocentese em 11 casos $(9,5 \%)$ não esteve significativamente associada ao parto vaginal em relação ao parto cesariana (Tabela 3). 
Dos 11 casos nos quais foi realizada cefalocentese, ocorreram complicações no parto em 6. Desses, 4 consistiram em dificuldade de extração fetal, em um caso houve prolapso de cordão e também ocorreu um caso de rotura uterina subclínica.

Tabela 3 - Forma de término do parto em casos de feto com hidrocefalia congênita conforme realização de cefalocentese.

\begin{tabular}{|c|c|c|}
\hline \multirow[t]{2}{*}{ Via de parto } & \multicolumn{2}{|c|}{ Cefalocentese } \\
\hline & Sim & Não \\
\hline Vaginal & 7 & 35 \\
\hline Cesárea & 4 & 66 \\
\hline Total & 11 & 101 \\
\hline
\end{tabular}

As complicações durante o parto ocorreram mais freqüentemente quando se optou pela via vaginal ( 15 de 42 partos) do que quando a resolução se deu por cesárea (12 de 70). Já as complicações puerperais ocorreram em $8,6 \%$ das pacientes, sem associação significativa com a via de parto (Tabela 4). Dos 82 casos com informações completas disponíveis sobre os recém-nascidos, o índice de Apgar aos 5 minutos foi significativamente mais elevado quando o parto foi cesariana (Tabela 5). A média de idade gestacional pelo índice de Capurro foi de 36,6 semanas e os recém-nascidos com hidrocefalia congênita pré-termo representaram $38,5 \%$ dos casos.

O perímetro cefálico variou de 26 a $57 \mathrm{~cm}$, com média de $36,1 \mathrm{~cm}$. A média de peso ao nascimento foi de $2.703 \mathrm{~g}$, tendo variado de $650 \mathrm{a}$ 4.960 g. A estatura variou de 33 a $56 \mathrm{~cm}$, com média de $46,5 \mathrm{~cm}$. A derivação ventricular foi realizada em 26 recém-nascidos, no $13^{\circ}$ dia de vida, em média. O diagnóstico morfogenético foi realizado em apenas um quarto dos casos (Tabela 6), entretanto várias outras anomalias congênitas estiveram associadas: persistência do canal arterial, comunicação interatrial, comunicação interventricular, atresia de esôfago, agenesia renal, espinha bífida, tetralogia de Fallot, microcefalia, fenda palatina, rins policísticos, hipoplasia pulmonar, malformações faciais, imperfuração anal, lábio leporino e atresia de cóanas.

A morte neonatal entre os recém-nascidos com hidrocefalia congênita ocorreu em 8 casos $(9,8 \%)$, restando 74 casos que foram acompanhados pelo Serviço de Neonatologia. As patologias mais freqüentes entre esses casos também são mostrados na Tabela 6 , embora várias outras condições patológicas tenham sido diagnosticadas, acometendo $73 \%$ dos recémnascidos vivos no período neonatal.
O tempo de internação neonatal variou de 1 a 158 dias, sendo, em média, de 30 dias. Durante o período de internação do recém-nascido, ocorreram mais 24 óbitos perinatais, num total de 32 , o que corresponde a uma mortalidade perinatal de 39\%. As condições clínicas dos recém-nascidos no momento da alta estão descritas na Tabela 7 , da mesma forma que a presença de seqüelas. Apenas uma pequena proporção do total de casos teve alta do berçário sem seqüelas.

Tabela 4 - Tipos de complicações no parto e no puerpério em mulheres com fetos com hidrocefalia, segundo a via de parto.

\begin{tabular}{|c|c|c|}
\hline \multirow[t]{2}{*}{ Complicações } & \multicolumn{2}{|c|}{ Via de parto } \\
\hline & Vaginal & Cesárea \\
\hline \multicolumn{3}{|l|}{ No parto ${ }^{a}$} \\
\hline Sem & 27 & 58 \\
\hline Dificuldade de extração & 8 & 9 \\
\hline Retenção placentária & 4 & 0 \\
\hline Prolapso de cordão & 1 & 1 \\
\hline Período expulsivo prolongado & 1 & 0 \\
\hline Hemorragia & 0 & 1 \\
\hline Distócia de ombro & 1 & 0 \\
\hline Rotura uterina subclinica & 0 & 1 \\
\hline \multicolumn{3}{|l|}{ Puerperais $^{\mathrm{b}}$} \\
\hline Sem & 38 & 64 \\
\hline Deiscência de sutura & 2 & 3 \\
\hline Endometrite puerperal & 1 & 2 \\
\hline Outras infecções puerperais* & 1 & 1 \\
\hline Total & 42 & 70 \\
\hline
\end{tabular}

* Mastite e/ou infecção do trato urinário

a Sem X qualquer $\chi^{2}=4,95 \quad \mathrm{p}=0,026$

${ }^{\mathrm{b}}$ Sem $\mathrm{X}$ qualquer Fisher $\quad \mathrm{p}=0,1$

Tabela 5 - Índice de Apgar aos 5 minutos de recém-nascidos com hidrocefalia congênita conforme a forma de término do parto.

\begin{tabular}{lcr} 
Apgar aos 5 min & \multicolumn{2}{c}{ Forma de término do parto } \\
\cline { 2 - 3 } & Vaginal & Cesárea \\
\hline Zero & 11 & 1 \\
1 a 6 & 5 & 8 \\
7 a 10 & 17 & 40 \\
Total & 33 & 49 \\
\hline & & $\mathrm{p}=0,0003$
\end{tabular}


Tabela 6 - Diagnóstico morfogenético, mortalidade e morbidade neonatal mais freqüentes na hidrocefalia congênita.

\begin{tabular}{lrr}
\hline & n & \% \\
\hline Diagnóstico morfogenético & & \\
$\quad$ Síndrome de Dandy-Walker & 6 & 7,3 \\
$\quad$ Síndrome de Arnold-Chiari & 5 & 6,1 \\
$\quad$ Síndrome de Patau & 1 & 1,2 \\
Síndrome de Edwards & 1 & 1,2 \\
Outras sindromes & 6 & 7,3 \\
& & \\
Morte neonatal & 8 & 9,8 \\
& & 100,0 \\
Total & 82 & \\
Morbidade neonatal & & 23,0 \\
Sepse & 17 & 12,2 \\
Incontinência urinária & 9 & 10,8 \\
Hipertensão intracraniana & 8 & 9,5 \\
Distúrbios metabólicos & 7 & 9,5 \\
Broncopneumonia & 7 & 8,1 \\
Incontinência fecal & 6 & 8,1 \\
Membrana hialina & 6 & 100,0 \\
Total & 74 & \\
\hline
\end{tabular}

Tabela 7 - Condições clínicas e seqüelas no momento da alta de recém-nascidos com hidrocefalia congênita.

\begin{tabular}{lrr} 
& $\mathbf{n}$ & $\%$ \\
\hline Condições clínicas na alta & & \\
$\quad$ Boa & 36 & 43,9 \\
Melhorado & 11 & 13,4 \\
$\quad$ Sem melhora & 1 & 1,2 \\
Óbito & 32 & 39,0 \\
Ignorado & 2 & 2,4 \\
Seqüelas & & \\
Sem seqüelas & 6 & 7,3 \\
Suspeito de seqüelas & 20 & 24,4 \\
$\quad$ Evidências de seqüelas & 22 & 26,8 \\
Óbito neonatal & 32 & 39,0 \\
Ignorado & 2 & 2,4 \\
Total & 82 & 100 \\
\hline
\end{tabular}

\section{Discussão}

Os dados do presente estudo demonstram que as mulheres com recém-nascidos hidrocéfalos são com freqüência jovens, de baixa paridade e com importante antecedente de aborto em sua história obstétrica pregressa. Na maioria dos casos, o diagnóstico foi realizado antes do parto, confirmado pelo exame ecográfico e o parto foi cesariana em quase dois terços dos casos. A cefalocentese foi realizada em 11 casos e as complicações no parto vaginal foram mais freqüentes que no parto por cesariana, ao passo que as complicações puerperais foram semelhantes. O índice de Apgar baixo foi mais freqüente entre os recém-nascidos de parto vaginal.
A hidrocefalia congênita esteve também associada a importante morbidade e mortalidade neonatal e perinatal, outras malformações, e com um número muito pequeno de crianças com alta hospitalar sem seqüelas.

Nenhuma gestante referiu ter usado droga ilícita (maconha, cocaina, etc.) e o medicamento mais utilizado foi um hipotensor, de uso mais frequente a partir do segundo trimestre da gestação e, sendo, portanto, pouco provável haver relação com o quadro apresentado pelo feto. Dentre os antecedentes obstétricos estudados, o abortamento foi o mais encontrado, em mais de um quarto dos casos.

Nesta casuística o diagnóstico da hidrocefalia ocorreu com mais freqüência durante a gestação e por meio de exame ultra-sonográfico. Há uma tendência mais recente de indicar a realização de cariótipo em amostra de sangue fetal obtido por cordocentese para diagnósticos genéticos mais precisos.

A complicação mais freqüente nos partos dessas crianças, como era de se esperar, foi a dificuldade de extração do pólo cefálico, presente tanto no parto vaginal quanto na cesariana. Podese admitir que a cefalocentese, enquanto procedimento para reduzir o diâmetro cefálico para a resolução do parto, seja mais utilizada nos casos de pior prognóstico, em que a via vaginal seria a escolhida. Entretanto, a opção pela forma de término do parto não se encontrou significativamente relacionada com a realização de cefalocentese, embora o parto vaginal tenha sido mais freqüente nesses casos. É provável que o pequeno número de procedimentos realizados não permita um poder estatístico para detectar tal diferença. Por outro lado, a cefalocentese durante uma cesariana também é compreensível, quando se pensa numa situação de prognóstico neonatal ruim e a possibilidade de graves lesões dos tecidos maternos durante a extração do pólo hidrocefálico. Além disso, a presença de outras anomalias congênitas associadas à hidrocefalia também é determinante do prognóstico perinatal e também da via de parto .

Apesar da vitalidade neonatal, medida pelo do índice de Apgar ao nascimento, ser pior entre os partos vaginais que entre as cesarianas, não é possivel estabelecer uma relação de causa e efeito entre essas duas variáveis. É razoável admitir que a gravidade da hidrocefalia diagnosticada e o pior prognóstico de evolução perinatal sejam mais determinantes da escolha do parto por via vaginal, e que essa gravidade é que seja a responsável pela baixa vitalidade neonatal, e não a via de parto.

Os resultados neonatais confirmam o esperado, ou seja, a grande morbidade e mortalidade associada à hidrocefalia congênita e às freqüentes outras malformações associadas. Cerca de $40 \%$ de todos os casos tiveram óbito perinatal e menos de $10 \%$ deles tiveram alta do berçário sem seqüelas detectáveis. Apesar do 
caráter retrospectivo do estudo, sem um protocolo assistencial previamente definido e com possíveis alterações de capacidade para o diagnóstico e de conduta ao longo deste período de dez anos, é possível dizer que o prognóstico perinatal de fetos com hidrocefalia, detectada tanto antes quanto depois do parto, é bastante reservado. Também é verdade que os modernos avanços tecnológicos, de diagnóstico por imagem e de procedimentos, utilizados atualmente pela Medicina Fetal, podem, a médio e a longo prazo, modificar o perfil diagnóstico, a terapêutica e o prognóstico desses recém-nascidos. Entretanto, essa não é a realidade atual para a maior parte da população brasileira que não dispõe desse tipo de atenção, a não ser em um muito pequeno número de hospitais universitários ou privados.

Para obstetras e pediatras, portanto, a criança com hidrocefalia representa um problema complexo, envolvendo múltiplos aspectos como diagnóstico etiológico, elaboração de um prognóstico e prevenção de sua recorrência, devendo-se lembrar ainda a importância do aconselhamento genético dos pais ${ }^{9}$.

A existência de malformação congênita durante a gestação é uma grande preocupação tanto para pais quanto para obstetras. Entre as malformações destaca-se a hidrocefalia por sua freqüência e letalidade, bem como pelo grande número de seqüelas que pode causar. O prognóstico de crianças nascidas com hidrocefalia depende, entre outros fatores, das condições de nascimento e da causa da hidrocefalia.Torna-se necessário, portanto, avaliar detalhadamente a evolução das gestações que cursam com hidrocefalia fetal, verificar suas complicações, dificuldades de condução e decisão sobre a via de parto.

\section{SUMMARY}

Objective: to evaluate the diagnosis, characteristics of pregnancy, maternal complications and perinatal outcome in cases of congenital hydrocephalus, and to associate them with pregnancy and delivery variables.

Methods: 116 pregnancies with this diagnosis were evaluated before or after delivery, 112 of them occurring at the Maternity ward of CAISM/UNICAMP during the period between 1986 and 1995. For perinatal variables, complete data of 82 newborns were used. For data analysis, distributions and means were calculated and $c^{2}$ and Fisher exact tests were applied.

Results: generally the diagnosis was made before delivery, confirmed by ultrasound and the delivery was through a cesarean section in cases. Cephalocentesis was performed in 11 cases and complications were more frequent in vaginal delivery than cesarean section. Low Apgar scores were more frequent among newborn babies delivered vaginally. Congenital hydrocephalus was also associated with important neonatal and perinatal morbidity and mortality, with other malformations, and a very low number of children without sequelae.

Conclusions: the evaluation of these factors may be of great value for the obstetrician who is following pregnant women with this fetal malformation. This could better support the decisions that, although medical and ethical, should take into account the risk-benefit ratio of measures to be taken.

KEY WORDS: Hydrocephalus. Prenatal diagnosis. Fetal malformation. Ultrasonography.

\section{Referências}

1.Arduini D, Rizzo G, Caforio L, Mancuso S. Development of behavioral states in hydrocephalic fetuses. Fetal Ther 1987; 2: 135-43.

2.Ceddia A, Di Rocco C, Iannelli A, Lauretti L. Idrocefalo neonatale ad eziologia non tumorale. Risultati del trattamento chirurgico nel primo mese di vita. Minerva Pediatr 1992; 44: 445-50.

3. Chervenak FA, Berkowitz RL, Romero R, Tortora M, Mayden K, Duncan C, et al. The diagnosis of fetal hydrocephalus. Am J Obstet Gynecol 1983; 147: 703-16.

4.Chervenak FA, Duncan C, Ment LR, Hobbins JC, McClure M, Scott D, et al. Outcome of fetal ventriculomegaly. Lancet 1984; 2: 179-81.

5.Chervenak FA, McCullough LB. Ethical analysis of the intrapartum management of pregnancy complicated by fetal hydrocephalus with macrocephaly. Obstet Gynecol 1986; 68: 720-5.

6.Cochrane DD, Myles ST, Nimrod C, Still DK, Sugarman RG, Wittmann BK, et al. Intrauterine hydrocephalus and ventriculomegaly: associated abnormalities and fetal outcome. Can $\mathbf{J}$ Neurol Sci 1985; 12: 51-9.

7.Cunningham FG, Williams JW, Gant NF, Leveno KJ, Gilstrap LC. Williams obstetrics. $19^{\text {th }}$ ed. Englewood Cliffs: Prentice Hall International; 1993.

8.Gandahusada S. Study on the prevalence of toxoplasmosis in Indonesia: a review. Southeast Asian J Trop Med Public Health 1991; 22(suppl): 93-8.

9.Habib Z. Genetics and genetic counselling in neonatal hydrocephalus. Obstet Gynecol Survy 1981; 36: 529-34. 
10. Matsushita H, Machado de Almeida GG. In: Diament AJ, Léfevre eds. Neurologia Infantil. $2^{\mathrm{a}}$ ed. Rio de Janeiro: Atheneu; 1989. p. 703-33.

11. Milhorat TH, Miller JI. Neurosurgery. In: Avery GB, Fletcher MA, MacDonald MG, editores. Neonatology: pathophysiology and management of the newborn. $4^{\text {th }}$ ed., J. B. Lippincott Co.; 1994.p. 1139-63.

12.Neme B. Distócia fetal. In: Neme B. Obstetrícia Básica. $1^{\mathrm{a}}$ ed.; São Paulo: Sarvier, p.1994.p.508- 537

13.Nyberg DA, Pretorius DH. Cerebral malformations. In: Diagnostic ultrassound of fetal anomalies - Text and Atlas. Nyberg DA, Mahony BS, Pretorius DH, editores. Year Book Medical Publishers, Inc. 1990.p.83-145,

14.Oi SZ, Yamada H, Kimura M, Ehara K, Matsumoto $\mathrm{S}$, Katayama $\mathrm{K}$ et al. Factors affecting prognosis of intrauterine hydrocephalus diagnosed in the third trimester-computerized data analysis on controversies in fetal surgery. Neurol Med Chir Tokyo 1990; 30: 456-461.

15. Serlo W, Kirkinen P, Jouppila P, Herva R. Prognostic signs in fetal hydrocephalus. Childs Nerv Syst 1986; 2: 93-97.

16.Shurtleff DB, Foltz ZL, Loeser JD. Hydrocephalus. A definition of its progression and relationship to intellectual function, diagnosis and complications. Am J Dis Child 1973; 125: 688-93.

17.Stoll C, Alembik Y, Dott B, Roth MP. An epidemiologic study of environmental and genetic factors in congenital hydrocephalus. Eur J Epidemiol 1992; 8: 797-803.

18.Vintzileos AM, Campbell WA, Weinbaum PJ, Nochimson DJ. Perinatal management and outcome of fetal ventriculomegaly. Obstet Gynecol 1987; 69: 5-11. 